\title{
Toxicidade de extratos de Anadenanthera macrocarpa (Fabaceae: Mimosoideae) e Bougainvillea buttiana (Nyctaginaceae) para lepidópteros-praga
}

\author{
Diego Tavares Carvalhinho ${ }^{1}$ \\ Raimundo Henrique Ferreira Rodrigues ${ }^{1}$ \\ Eliane Carneiro $^{2}$ \\ Gleidyane Novais Lopes ${ }^{1 *}$ \\ Luciana Barboza Silva ${ }^{1}$ \\ Andrea Cristiane Baptistel ${ }^{1}$ \\ Bruno Ettore Pavan ${ }^{3}$ \\ ${ }^{1}$ Universidade Federal do Piauí, Bom Jesus, Campus Professora Cinobelina Elvas \\ CEP 6490000, Bom Jesus - PI, Brasil \\ ${ }^{2}$ Universidade Tecnológica Federal do Paraná, Pato Branco - PR, Brasil \\ ${ }^{3}$ Faculdade de Engenharia UNESP, Ilha Solteira - SP, Brasil \\ * Autor para correspondência \\ gnlopesm@hotmail.com
}

Submetido em 01/06/2016

Aceito para publicação em 01/03/2017

\section{Resumo}

O uso de extratos vegetais apresenta grande eficiência no manejo de pragas, destinados a produções agrícolas de pequeno e médio porte, por controlar uma gama muito grande de artrópodes. O objetivo desta pesquisa consiste em avaliar os efeitos tóxicos dos extratos de Anadenanthera macrocarpa (Fabaceae: Mimosoideae) e Bougainvillea buttiana (Nyctaginaceae) em Helicoverpa armigera (Hübner), Spodoptera cosmioides (Walker) e Spodoptera frugiperda (Smith) (Lepidoptera: Noctuidae). Lagartas de H. armigera e S. cosmioides foram mantidas em discos foliares de soja, enquanto $S$. frugiperda foi mantida em discos foliares de milho, tratados com os extratos aquoso diluídos nas concentrações de: $0,500,1.000,2.500,4.000,5.000,10.000$ e 15.000 mg.L-1. Foram mensurados os efeitos letais e subletais dos extratos sobre os insetos. De acordo com os resultados obtidos, a concentração de $500 \mathrm{mg} . \mathrm{L}^{-1}$ do extrato de $A$. macrocarpa causou o maior percentual de mortalidade $(93,33 \%) \mathrm{em} \mathrm{H}$. armigera. O extrato de $B$. buttiana causou $86,67 \%$ e $60 \%$ de mortalidade em $H$. armigera $\left(1.000 \mathrm{mg} . \mathrm{L}^{-1}\right)$ e $S$. cosmioides (4.000 mg. $\mathrm{L}^{-1}$ ), respectivamente. A redução da emergência dos adultos foi o principal efeito subletal constatado em S. cosmioides e S. frugiperda. Considerando os resultados obtidos, destaca-se que os extratos vegetais utilizados apresentam-se como uma alternativa em potencial para o manejo de lepidópteros-praga.

Palavras-chave: Angico-preto; Lagartas desfolhadoras; Três-marias

\section{Abstract}

Toxicity of Anadenanthera macrocarpa (Fabaceae: Mimosoideae) and Bougainvillea buttiana (Nyctaginaceae) extracts to lepidopteran pests. For small and medium agricultural production, vegetal extracts 
are highly efficient at managing pests because they control a wide range of arthropods. The objective of this research was to evaluate the toxic effects of Anadenanthera macrocarpa (Fabaceae: Mimosoideae) and Bougainvillea buttiana (Nyctaginaceae) extracts on Helicoverpa armigera (Hübner), Spodoptera cosmioides (Walker) and Spodoptera frugiperda (Smith) (Lepidoptera: Noctuidae). Helicoverpa armigera and S. cosmioides caterpillars were maintained on soybean leaf discs, and $S$. frugiperda was maintained on corn leaf discs, treated with diluted aqueous extracts at concentrations of $0,500,1,000,2,500,4,000,5,000,10,000$ and 15,000 mg.L. ${ }^{-1}$. The lethal and sublethal effects of the extracts on the insects were measured. According to the results, the concentration of 500 mg.L $\mathrm{L}^{-1}$ of the $A$. macrocarpa extract caused the highest percentage of mortality $(93.33 \%)$ of $H$. armigera. The extract of B. buttiana caused $86.67 \%$ and $60 \%$ mortality of $H$. armigera $\left(1,000 \mathrm{mg} \cdot \mathrm{L}^{-1}\right)$ and S. cosmioides $\left(4,000 \mathrm{mg} . \mathrm{L}^{-1}\right)$, respectively. The reduction of the emergence of adults was the main sublethal effect found for S. cosmioides and S. frugiperda. Considering the results, the vegetal extracts used in this research are a potential alternative for the management of lepidopteran pests.

Key words: Black mimosa; Caterpillars defoliating; Three Marys

\section{Introdução}

O cultivo de grãos sofre com ataques constantes de lagartas desfolhadoras, como Helicoverpa armigera (Hübner), Spodoptera cosmioides (Walker) e Spodoptera frugiperda (Smith) (Lepidoptera: Noctuidae) (CABEZAS et al., 2013; CZEPAK et al., 2013). Essas espécies apresentam uma grande importância agronômica em decorrência do elevado potencial de dano em virtude de características como polifagia e ciclo biológico curto. O potencial de dano dessas espécies, conciliado com o uso indiscriminado ou inadequado de produtos químicos e plantas transgênicas, tem propiciado grandes perdas agrícolas, além de resultar na redução da eficiência das tecnologias de manejo empregadas (AHMAD, 2009; WANG et al., 2010; LEITE et al., 2016).

O uso de extratos vegetais na agricultura apresentase como uma alternativa viável e eficiente no controle de pragas, pois um grande número de moléculas químicas inseticidas sintéticas foi moldado a partir de substâncias orgânicas provenientes de espécies vegetais. Como exemplo disso, têm-se os carbamatos análogos à fisostigmina, uma substância presente na semente de Physostigma venenosum. (Fabaceae) (TRIGGLE et al., 1998; ZHAO et al., 2004), os piretroides análogos à piretrina, presente nas flores de Chrysanthemum cinerariaefolium (Asteraceae) (KATSUDA, 1999), e a reanodina, proveniente de Ryania speciosa (Flacourtiaceae) (CORONADO et al., 1994; EL-WAKEIL, 2013).

Uma ferramenta alternativa e sustentável de controle de pragas consiste na identificação de novos compostos botânicos bioativos compatíveis com os programas de manejo de pragas (MEDEIROS et al., 2005), que apresentem específico espectro e modo de ação e que sejam fácil de processar e aplicar (EL-WAKEIL, 2013; DINESH et al., 2014). As moléculas inseticidas encontradas nas plantas são oriundas principalmente da síntese de metabólitos secundários dos vegetais, presentes em famílias como Asteraceae, Fabaceae, Malvaceae e Meliaceae (VARMA; DUBEY, 1999; VIEGAS-JÚNIOR, 2003; DINESH et al., 2014), sendo constatado, nos últimos anos, um aumento crescente na identificação de espécies vegetais com potencial inseticida.

Os principais metabólitos secundários presentes em espécies de Fabaceae e Nyctaginaceae são: terpenos, flavonoides, alcaloides e taninos. Sendo que elevadas concentrações de inibidores de protease e tripsina estão presentes nas fabáceas (CHAN et al., 2013). Estudos demonstram que o extrato de Adenanthera pavonina (Fabaceae) tem efeito letal em Diatraea saccharalis (Fabricius) (Lepidoptera: Pyralidae) (SILVA et al., 2012), assim como o extrato de Bougainvillea spp. (Nyctaginaceae) em espécies de lepidópteros-praga, incluindo $H$. armigera (BOIÇA JÚNIOR et al., 2005; LODHA et al., 2011).

Nesse contexto, trabalhos que visem identificar plantas endêmicas com ação inseticida embasados nos avanços e perspectivas relacionadas ao manejo de lepidópteros praga apresentam grande relevância, sendo o objetivo desta pesquisa avaliar os efeitos tóxicos letais e subletais dos extratos obtidos a partir de Anadenanthera macrocarpa e Bougainvillea buttiana em $H$. armigera, $S$. frugiperda e $S$. cosmioides. 


\section{Material e Métodos}

\section{Local do experimento}

O experimento foi conduzido no Laboratório de Fitotecnia da Universidade Federal do Piauí, Campus Professora Cinobelina Elvas (CPCE/UFPI) no período de agosto de 2014 a julho de 2015.

\section{Criação dos insetos}

As lagartas foram coletadas na região do alto médio Gurgueia Bom Jesus - PI, em lavouras de cultivo intensivo de soja e milho, no início da safra 2014/2015. Nos bioensaios foram utilizadas as espécies $H$. armigera, $S$. cosmioides e $S$. frugiperda, mantidas na criação do Laboratório de Fitotecnia (CPCE/UFPI), em condições controladas $\left(25 \pm 5^{\circ} \mathrm{C}, 60 \pm 10 \%\right.$ UR, 12:12 LD). Os adultos foram acondicionados em gaiolas de PVC (30 x $20 \mathrm{~cm} \varnothing)$, alimentados com solução de mel a 10\%. A cada dois dias foram coletadas as posturas e acondicionadas em copos plásticos até a eclosão das lagartas das três espécies estudadas, as quais foram transferidas para a dieta artificial adaptada de Kasten Jr. et al. (1978). A confirmação específica de $H$. armigera foi realizada pelo pesquisador Dr. Sergio Ide, do Instituto Biológico, SP.

\section{Material vegetal}

Foram elaborados dois tipos de extrato aquoso (EA), sendo um de folhas e cascas do caule de Bougainvillea buttiana e outro de raízes de $A$. macrocarpa. Os materiais vegetais foram coletados no município de Bom Jesus, PI. O material vegetal foi cortado em pedaços menores e submetido à secagem em estufa de circulação forçada de ar, até as amostras apresentarem massa constante, por aproximadamente $72 \mathrm{~h}$, em temperatura entre 40-50 ${ }^{\circ} \mathrm{C}$. Após a completa secagem, triturou-se o material em moinho, que foi acondicionado em um frasco de vidro âmbar hermeticamente fechado. O material testemunha foi herborizado, processado segundo técnicas usuais (MORI et al., 1989) e incluído no acervo do Herbário Setorial Vale do Gurgueia (Número de registro - 69; Ficheiro - 02) da Universidade Federal do Piauí, vinculado ao Herbário Institucional Graziela Barroso (TEPB/UFPI).

\section{Preparo do extrato aquoso}

Para o preparo dois tipos de extrato aquoso (EA) de A. macrocarpa e B. buttiana, $60 \mathrm{~g}$ do material vegetal seco foram submetidos à decocção de 2 minutos, empregando $540 \mathrm{~mL}$ de água destilada, cujo rendimento foi de $450 \mathrm{~mL}$ de extrato após decocção de 2 minutos. Retiraram-se três alíquotas de $1 \mathrm{~mL}$ da solução obtida para determinar a massa seca do extrato. As alíquotas foram acondicionadas em frascos lavados, secos, desengordurados, previamente pesados e identificados, os quais foram colocados em um dessecador até a obtenção de uma massa constante para cada frasco. A massa média obtida referente a $1 \mathrm{~mL}$ da solução foi relacionada ao respectivo volume total, obtendo-se o extrato bruto em mg.mL $\mathrm{mL}^{-1}$.

\section{Bioensaio}

Utilizou-se o extrato de $A$. macrocarpa e $B$. buttiana nas concentrações, 500 1.000, 2.500, 4.000, 5.000, 10.000 e $15.000 \mathrm{mg} . \mathrm{L}^{-1}$ da solução diluída em álcool etílico $99,3 \%$. Folhas frescas de soja e milho foram coletadas, do terço superior de plantas cultivadas em vasos, para a obtenção dos discos foliares ( $5 \mathrm{~cm} \varnothing)$. Os discos foliares foram mergulhados nas referidas concentrações por 10 segundos com agitação suave e secos ao ar durante 30 minutos, à temperatura ambiente. Discos de soja foram oferecidos para alimentação das lagartas de 3ํㅡㄹ instar (cinco dias) de $H$. armigera e $S$. cosmioides, e discos de milho para $S$. frugiperda. Utilizaram-se discos de folhas de soja e milho mergulhados apenas em álcool etílico $99,3 \%$ como testemunha. A terceira geração das lagartas foi utilizada na montagem do experimento.

\section{Delineamento experimental}

O delineamento experimental utilizado foi o inteiramente casualizado, com sete tratamentos (concentrações), três repetições (10 lagartas/repetição), além da testemunha. As lagartas foram mantidas expostas aos discos foliares tratados, individualizadas em copos plásticos de $200 \mathrm{~mL}$ em condições laboratório $\left(25 \pm 5^{\circ} \mathrm{C}, 60 \pm 10 \% \mathrm{UR}, 12: 12 \mathrm{LD}\right)$ por um período de $120 \mathrm{~h}$, os discos foliares foram trocados a cada 24 h. A mortalidade das lagartas foi avaliada após 
$120 \mathrm{~h}$ de exposição aos extratos. Foram consideradas mortas as lagartas que após estimulo físico com pinça não apresentaram movimentos. Após esse período, as lagartas sobreviventes foram alimentadas com dieta artificial para observação dos efeitos subletais. A toxicidade dos extratos sobre as lagartas $H$. armigera, $S$. cosmioides, $S$. frugiperda foi avaliada em função dos parâmetros: mortalidade, massa das larvas e pupas e emergência dos adultos. O peso larval foi avaliado após $120 \mathrm{~h}$ de exposição aos extratos. A massa das pupas foi avaliada em no máximo 24 h após a mudança de estágio. As concentrações letais (CL) foram determinadas apenas para a espécie $H$. armigera, pois os dados referentes às demais espécies não se adequaram ao modelo Probit.

\section{Análise dos dados}

Os dados referentes aos parâmetros avaliados foram analisados estatisticamente no modelo fatorial
$2 \times 8$ (dois produtos e oito doses), comparados entre si pelo teste Tukey a 5\% de significância, programa $R$ Version 3.2.1 (R CORE TEAM, 2015), os dados foram submetidos ao teste de normalidade Shapiro-Wilk (W) e transformados para $\sqrt{(x+0,5)}$ quando necessário. As concentrações letais (CL 50 e 95\%) foram calculadas pela rotina Probit, com o programa estatístico SAS (SAS INSTITUTE, 2002).

\section{Resultados}

Os resultados obtidos no bioensaio de mortalidade apresentaram interação entre extrato e dose, para as três espécies de lepidópteros avaliados. Helicoverpa armigera apresentou mortalidade após exposição aos extratos (Tabela 1), em que a concentração de 500 mg.L. $\mathrm{L}^{-1}$ do extrato de $A$. macrocarpa resultou em maior mortalidade (93,33\%). O extrato de Bougainvillea buttiana causou maior mortalidade $(86,67 \%)$ em

TABELA 1: Mortalidade acumulada ( \pm EP) de lepidópteros desfolhadores após $120 \mathrm{~h}$ de exposição aos extratos de Anadenanthera macrocarpa (Fabaceae: Mimosoideae) e Bougainvillea buttiana (Nyctaginaceae).

\begin{tabular}{|c|c|c|}
\hline \multicolumn{3}{|c|}{ Mortalidade $(\%)^{1}$} \\
\hline Conc. (mg.L.-1) & A. macrocarpa & B. buttiana \\
\hline \multicolumn{3}{|l|}{ Helicoverpa armigera } \\
\hline Controle (etanol) & $0,00 \pm 0,00 \mathrm{Ea}$ & $0,00 \pm 0,00 \mathrm{Da}$ \\
\hline 500 & $93,33 \pm 3,65 \mathrm{Aa}$ & $10,00 \pm 3,16 \mathrm{Db}$ \\
\hline 1.000 & $66,67 \pm 3,65 \mathrm{Aba}$ & $86,67 \pm 1,83 \mathrm{Aa}$ \\
\hline 2.500 & $36,67 \pm 2,58 \mathrm{Bbcd}$ & $80,00 \pm 0,00 \mathrm{Aba}$ \\
\hline 4.000 & $46,67 \pm 1,83 \mathrm{BCa}$ & $50,00 \pm 3,16 \mathrm{BCa}$ \\
\hline 5.000 & $63,33 \pm 1,83 \mathrm{ABCa}$ & $33,31 \pm 1,83 \mathrm{Cb}$ \\
\hline 10.000 & $23,33 \pm 6,58 \mathrm{Db}$ & $56,67 \pm 1,83 \mathrm{ABCa}$ \\
\hline 15.000 & $33,33 \pm 1,83 \mathrm{CDa}$ & $36,67 \pm 3,65 \mathrm{Ca}$ \\
\hline $\mathrm{F}$ & \multicolumn{2}{|c|}{$1911 * *$} \\
\hline C.V. $(\%)$ & \multicolumn{2}{|c|}{12,37} \\
\hline \multicolumn{3}{|l|}{ Spodoptera cosmioides } \\
\hline Controle (etanol) & $3,33 \pm 1,83 \mathrm{Aa}$ & $0,00 \pm 0,00 \mathrm{Ca}$ \\
\hline 500 & $13,33 \pm 3,65 \mathrm{Aa}$ & $0,00 \pm 0,00 \mathrm{Cb}$ \\
\hline 1.000 & $13,33 \pm 1,83 \mathrm{Aa}$ & $10,00 \pm 0,00 \mathrm{BCa}$ \\
\hline 2.500 & $3,33 \pm 1,83 \mathrm{Aa}$ & $3,33 \pm 1,83 \mathrm{Ca}$ \\
\hline 4.000 & $13,33 \pm 1,83 \mathrm{Ab}$ & $60,00 \pm 1,30 \mathrm{Aa}$ \\
\hline 5.000 & $13,33 \pm 1,83 \mathrm{Ab}$ & $30,00 \pm 1,20 \mathrm{Aba}$ \\
\hline 10.000 & $6,67 \pm 1,83 \mathrm{Aa}$ & $3,33 \pm 1,83 \mathrm{Ca}$ \\
\hline 15.000 & $20,00 \pm 5,48 \mathrm{Aa}$ & $23,33 \pm 1,83 \mathrm{Ba}$ \\
\hline $\mathrm{F}$ & \multicolumn{2}{|c|}{$5,47 * *$} \\
\hline C.V. $(\%)$ & \multicolumn{2}{|c|}{22,74} \\
\hline
\end{tabular}




\begin{tabular}{lcc}
\hline Spodoptera frugiperda & & \\
\hline Controle (etanol) & $0,00 \pm 0,00 \mathrm{Aa}$ & $0,00 \pm 0,00 \mathrm{Ba}$ \\
500 & $0,00 \pm 0,00 \mathrm{Aa}$ & $0,00 \pm 0,00 \mathrm{Ba}$ \\
1.000 & $0,00 \pm 0,00 \mathrm{Ab}$ & $10,00 \pm 0,00 \mathrm{Aa}$ \\
2.500 & $0,00 \pm 0,00 \mathrm{Aa}$ & $3,33 \pm 1,18 \mathrm{ABa}$ \\
4.000 & $0,00 \pm 0,00 \mathrm{Ab}$ & $10,00 \pm 0,00 \mathrm{Aa}$ \\
5.000 & $3,33 \pm 0,18 \mathrm{Ab}$ & $10,00 \pm 0,00 \mathrm{Aa}$ \\
10.000 & $0,00 \pm 0,00 \mathrm{Aa}$ & $0,00 \pm 0,00 \mathrm{Ba}$ \\
15.000 & $0,00 \pm 0,00 \mathrm{Aa}$ & $0,00 \pm 0,00 \mathrm{Ba}$ \\
\hline F & & \\
C.V. $(\%)$ & $323^{* *}$ & \\
\hline
\end{tabular}

${ }^{1}$ Médias seguidas pela mesma letra, minúsculas na linha e maiúsculas na coluna, não diferem entre si pelo teste Tukey a $5 \%$ de probabilidade. Os valores foram transformados para $\sqrt{(x+0,5)}$.

TABELA 2: Concentração letal do extrato de Anadenanthera macrocarpa (Fabaceae: Mimosoideae) a Helicoverpa armigera.

\begin{tabular}{|c|c|c|c|c|c|}
\hline \multirow[b]{2}{*}{ Tratamento } & \multicolumn{2}{|c|}{ Concentração Letal $\left(\mathrm{Lha}^{-1}\right)$} & \multirow[b]{2}{*}{$\begin{array}{c}\text { Inclinação } \\
\pm \text { DP }\end{array}$} & \multirow[b]{2}{*}{$X^{2}$} & \multirow[b]{2}{*}{$p$} \\
\hline & $\begin{array}{c}\mathrm{CL}_{50} \\
\text { (IC } 95 \%)\end{array}$ & $\begin{array}{c}\mathrm{CL}_{95} \\
\text { (IC 95\%) }\end{array}$ & & & \\
\hline $\begin{array}{c}\text { Anadenanthera } \\
\text { macrocarpa }\end{array}$ & $\begin{array}{c}3,73 \\
(2,49-5,68)\end{array}$ & $\begin{array}{c}108,84 \\
(14,15-290,61)\end{array}$ & $-1,07 \pm 0,19$ & 22,93 & $0,24 * *$ \\
\hline
\end{tabular}

D.P. desvio padrão; $X^{2}=$ qui-quadrado; IC $=$ intervalo de confiança; $p=$ probabilidade. ${ }^{*} *$ Significativo a 0,01 .

H. armigera nas concentrações de $1.000 \mathrm{mg} . \mathrm{L}^{-1}$ (Tabela 2).

O extrato de Bougainvillea buttiana causou mortalidade de $60 \%$ em $S$. cosmioides na concentração de $4.000 \mathrm{mg} \cdot \mathrm{L}^{-1}$ (Tabela 1). No entanto os extratos proporcionaram um baixo percentual de mortalidade em S. frugiperda, a concentração de $5.000 \mathrm{mg}$.L${ }^{1}$ provocou o maior percentual de mortalidade de $3,33 \%$ e $10 \%$ para os extratos de $A$. macrocarpa e Bougainvillea buttiana, respectivamente (Tabela 1).

Os elevados percentuais de mortalidade de $H$. armigera após a exposição ao extrato de $A$. macrocarpa permitiram a utilização da rotina Probit. Os resultados referentes à mortalidade dos outros tratamentos foram inferiores a $90 \%$, o que pode ter inviabilizado a utilização da análise de Probit e a determinação das concentrações letais (CL50 e CL95). Determinou-se que a concentração letal para matar $50 \%$ da população (CL50) de $H$. armigera foi de 3,73 L.ha-1 e a CL95 correspondendo a 108,84 L.ha-1 (Tabela 2), os baixos valores de quiquadrado nos bioensaios indicam uma homogeneidade da população teste.

Os efeitos subletais não foram estabelecidos para H. armigera em função do baixo número de indivíduos sobreviventes ao final do ciclo larval. O resumo da análise de variância para os parâmetros biológicos avaliados está descrito na Tabela 3. O peso larval e a viabilidade das pupas de $S$. cosmioides foram alterados pela ação dos extratos, bem como a viabilidade das pupas de $S$. frugiperda (Tabela 3). A concentração de 2.500 mg. $L^{-1}$ do extrato de $B$. buttiana reduziu o peso larval em 24,86\% (7,80 mg), em relação à testemunha, para $S$. cosmioides (Tabela 4). Não foram constatadas alterações na viabilidade larval em $S$. cosmioides e $S$. frugiperda. Os extratos não apresentaram efeitos sobre a massa das pupas de $S$. cosmioides e $S$. frugiperda (Tabela 4). Foi constada uma redução na emergência das espécies estudadas (Tabela 5). 
TABELA 3: Análise da variância dos parâmetros biológicos de Spodoptera cosmioides e Spodoptera frugiperda após $120 \mathrm{~h}$ de exposição aos extratos de Anadenanthera macrocarpa (Fabaceae: Mimosoideae) e Bougainvillea buttiana (Nyctaginaceae).

\begin{tabular}{lccccc}
\hline \multirow{2}{*}{$\begin{array}{l}\text { Fontes de } \\
\text { Variação }\end{array}$} & $\begin{array}{c}\text { Graus de } \\
\text { Liberdade }\end{array}$ & Peso larval & Viabilidade larval & Peso das pupas & $\begin{array}{c}\text { Emergência dos } \\
\text { adultos }\end{array}$ \\
\cline { 3 - 5 } & \multicolumn{5}{c}{ Spodoptera cosmioides } \\
\hline Extrato (e) & 1 & $345,82^{* *}$ & $174,75^{\text {N.S. }}$ & $128,62^{\text {N.S. }}$ & $748,00^{*}$ \\
Conc. (a) & 7 & $28,62^{*}$ & $393,60^{\text {N.S. }}$ & $1509,68^{*}$ & $896,76^{* *}$ \\
ExA & 7 & $40,92^{* *}$ & $300,38^{\text {N.S. }}$ & $474,27^{\text {N.S. }}$ & $705,71^{* *}$ \\
Erro & 32 & 9,11 & 198,00 & 484,726 & 127,56 \\
Média Geral & - & 12,20 & 85,61 & 478,82 & 77,62 \\
C.V.\% & - & 24,73 & 16,44 & 4,60 & 14,55 \\
\hline & 1 & Spodoptera frugiperda & & $4,68^{\text {N.S. }}$ \\
\hline Extrato (e) & 7 & $73,67^{\text {N.S. }}$ & $83,56^{\text {N.S. }}$ & $239,02^{\text {N.S. }}$ & $221,36^{* *}$ \\
Conc. (a) & 7 & $73,67^{*}$ & $57,28^{*}$ & $236,57^{*}$ & $140,81^{*}$ \\
ExA & 32 & $37,82^{\text {N.S. }}$ & $43,23^{\text {N.S. }}$ & $144,81^{\text {N.S. }}$ & 47,10 \\
Erro & 18,97 & 23,27 & 101,57 & 92,18 \\
Média Geral & - & 26,80 & 97,80 & 232,99 & 7,44 \\
C.V.\% & - & 16,25 & 4,93 & 4,33 & \\
\hline
\end{tabular}

**Significativo a 0,$01 ;$ *Significativo a 0,05 . N.S. Não significativo.

TABELA 4: Peso larval (mg) de Spodoptera cosmioides após 120 h de exposição aos extratos de Anadenanthera macrocarpa (Fabaceae: Mimosoideae) e Bougainvillea buttiana (Nyctaginaceae).

\begin{tabular}{|c|c|c|}
\hline \multirow{2}{*}{ Concentração $\left(\mathrm{mg}^{-\mathrm{L}^{-1}}\right)$} & \multicolumn{2}{|c|}{ Peso larval (mg) $^{1}$} \\
\hline & A. Macrocarpa & B. buttiana \\
\hline Controle & $10,38 \pm 0,34 \mathrm{aC}$ & $10,15 \pm 0,20 \mathrm{aA}$ \\
\hline 500 & $16,88 \pm 2,23 \mathrm{aABC}$ & $10,39 \pm 0,38 \mathrm{bA}$ \\
\hline 1.000 & $22,19 \pm 2,70 \mathrm{aA}$ & $9,38 \pm 1,45 \mathrm{bA}$ \\
\hline 2.500 & $20,77 \pm 3,40 \mathrm{aAB}$ & $7,80 \pm 1,30 \mathrm{bA}$ \\
\hline 4.000 & $14,85 \pm 2,08 \mathrm{aABC}$ & $10,28 \pm 1,98 \mathrm{aA}$ \\
\hline 5.000 & $12,94 \pm 1,71 \mathrm{aBC}$ & $8,34 \pm 0,84 \mathrm{aA}$ \\
\hline 10.000 & $9,95 \pm 0,78 \mathrm{aC}$ & $10,75 \pm 2,22 \mathrm{aA}$ \\
\hline 15.000 & $11,14 \pm 1,27 \mathrm{aC}$ & $9,08 \pm 1,28 \mathrm{aA}$ \\
\hline
\end{tabular}

As concentrações de $4.000 \mathrm{mg} . \mathrm{L}^{-1}$ do extrato de A. macrocarpa e $5.000 \mathrm{mg} . \mathrm{L}^{-1}$ de $B$. buttiana reduziram a emergência de adultos de $S$. cosmioides em 31,48\% e $72,22 \%$, respectivamente (Tabela 5). Para a $S$. frugiperda, a concentração de $5.000 \mathrm{mg} \cdot \mathrm{L}^{-1}$ reduziu em $20 \%$ a emergência dos adultos em relação à testemunha, para ambos os extratos. 
TABELA 5: Emergência dos adultos de Spodoptera cosmioides e Spodoptera frugiperda após exposição aos extratos de Anadenanthera macrocarpa (Fabaceae: Mimosoideae) e Bougainvillea buttiana (Nyctaginaceae).

\begin{tabular}{lcc}
\hline \multicolumn{1}{c}{ Concentração (mg.L $\left.{ }^{-1}\right)$} & \multicolumn{2}{c}{ Emergência dos adultos $(\%)^{\mathbf{1}}$} \\
\hline Spodoptera cosmioides & A. macrocarpa & B. buttiana \\
Controle (etanol) & $100.00 \pm 0 \mathrm{aA}$ & $100.00 \pm 0 \mathrm{aA}$ \\
500 & $70.00 \pm 8.78 \mathrm{aB}$ & $84.44 \pm 2.94 \mathrm{aAB}$ \\
1.000 & $82.74 \pm 3.90 \mathrm{aAB}$ & $74.60 \pm 5.72 \mathrm{aAB}$ \\
2.500 & $79.17 \pm 5.07 \mathrm{aAB}$ & $74.60 \pm 5.72 \mathrm{aAB}$ \\
4.000 & $68.52 \pm 8.07 \mathrm{aB}$ & $70.00 \pm 10.00 \mathrm{aB}$ \\
5.000 & $85.52 \pm 1.21 \mathrm{aAB}$ & $27.78 \pm 5.56 \mathrm{bC}$ \\
10.000 & $81.48 \pm 1.85 \mathrm{aAB}$ & $85.00 \pm 7.64 \mathrm{aAB}$ \\
15.000 & $85.19 \pm 9.80 \mathrm{aAB}$ & $73.02 \pm 11.53 \mathrm{aAB}$ \\
\hline Spodoptera frugiperda & & \\
\hline Controle (etanol) & $100,00 \pm 0 \mathrm{aA}$ & $100,00 \pm 0,00 \mathrm{aA}$ \\
500 & $89,63 \pm 0,37 \mathrm{aAB}$ & $100,00 \pm 0,00 \mathrm{aA}$ \\
1.000 & $100,00 \pm 0,00 \mathrm{aA}$ & $86,67 \pm 3,33 \mathrm{bAB}$ \\
2.500 & $82,22 \pm 3,39 \mathrm{~b} \mathrm{AB}$ & $96,67 \pm 3,33 \mathrm{aAB}$ \\
4.000 & $100,00 \pm 0,00 \mathrm{aA}$ & $90,00 \pm 5,77 \mathrm{aAB}$ \\
5.000 & $80,56 \pm 4,24 \mathrm{aB}$ & $80,00 \pm 5,77 \mathrm{aB}$ \\
10.000 & $92,59 \pm 7,41 \mathrm{aAB}$ & $100,00 \pm 0,00 \mathrm{aA}$ \\
15.000 & $90,00 \pm 5,77 \mathrm{aAB}$ & $86,67 \pm 6,67 \mathrm{aAB}$ \\
\hline
\end{tabular}

${ }^{1}$ Médias seguidas pela mesma letra, minúsculas na linha e maiúsculas na coluna, não diferem entre si pelo teste Tukey a $5 \%$ de probabilidade.

\section{Discussão}

Todas as concentrações dos extratos utilizados apresentaram toxicidade para $H$. armigera. Estudos realizados com extrato de Hygrophila schulii (BuchHam.) (Syn. H. auriculata) (Acanthaceae) (BASKAR et al., 2015) e com extrato de Clerodendron infortunatum Linn. (Verbenaceae) (ABBASZADEH et al., 2014), sobre $H$. armigera com concentrações variando de 5.000 a $50.000 \mathrm{mg} . \mathrm{L}^{-1}$, apresentaram percentuais de mortalidade semelhantes aos obtidos no presente estudo. Provavelmente os elevados índices de mortalidade constatados após a exposição de $H$. armigera aos extratos vegetais podem estar atrelados à presença de fitoquímicos com atividade inseticida, como os terpenoides, flavonoides e esteroides (WEBER et al., 2011), presentes em elevadas concentrações nas famílias vegetais estudadas, assim como em Fabaceae (CHAN et al., 2013), Asteraceae (RIBEIRO et al., 2010) e Verbaceae (PARRA-GARCÉS et al., 2010). Frações do extrato de Couroupita guianensis (Aubl) (Lecythidaceae) também apresentam efeitos tóxicos em $H$. armigera, obtendo valores de $\mathrm{CL}_{50}$ de 2,$72 ; 5.21 \mathrm{e}$
7,22 para os extratos de hexano, clorofórmio e acetato de etila, respectivamente (BASKAR et al., 2010). A toxicidade do extrato bruto de $A$. macrocarpa ocasionou $80 \%$ de mortalidade para imaturos de Rhipicephalus (Boophilus) microplus (Acari; Ixodidae) (SILVA FILHO et al., 2013), demonstrando que o extrato bruto utilizado no experimento apresenta toxicidade similar a extratos fracionados, e ainda que controla outros grupos de artrópodes, podendo ser empregado no manejo de diferentes pragas.

A identificação e quantificação dos fitoquímicos presentes nos extratos devem ser estudados em trabalhos futuros a fim de conhecer os mecanismos envolvidos na ação inseticida dos extratos. Figueredo et al. (2013) identificaram os compostos potencialmente bioativos presentes em $A$. macrocarpa, como fenóis, taninos pirogalatos, taninos-flobafenos, antocianinas, antocianidinas, flavonas, flavonóis, xantonas, chalconas, auronas, flavononóis, leucoantocianidinas, catequinas, flavononas e alcaloides. Esses compostos alteram a sinapse nervosa, respiração, equilíbrio hormonal, reprodução e comportamento, reduzindo a viabilidade 
larval, emergência dos adultos e aumentando o tempo para a taxa de crescimento da população (VARMA; DUBEY, 1999; ABBASZADEH et al., 2014; DINESH et al., 2014; BASKAR et al., 2015).

A mortalidade obtida com extrato de B. buttiana sobre $H$. armigera e $S$. cosmioides foi superior à constatada por Lodha et al. (2011) em seu experimento com efeito letal e subletal do extrato de $B$. buttiana na dieta sobre $H$. armigera, em que a concentração de 33,33 mg. $\mathrm{L}^{-1}$ proporcionou mortalidade máxima de $50 \%$. Boiça Júnior et al. (2005), em seu experimento com efeito letal do extrato de Bougainvillea glabra sobre Plutella xylostella (Linnaeus, 1758) (Lepidoptera: Plutellidae), constataram que a concentração de $10 \%$ (100.000 mg. $\mathrm{L}^{-1}$ ) provocou $72,9 \%$ de mortalidade. Contudo o método de extração realizado por Boiça Júnior et al. (2005) foi semelhante ao realizado neste experimento, evidenciando que o extrato utilizado foi mais tóxico do que outros elaborados da mesma espécie vegetal. Os resultados obtidos permitem estabelecer que extrato de $B$. buttiana apresenta potencial tóxico sobre lepidópteros-praga de importância econômica.

As menores concentrações dos extratos provocaram os maiores percentuais de mortalidade nas lagartas de $H$. armigera e $S$. cosmioides, ocorrendo uma inversão na relação concentração-resposta (mortalidade), sendo conhecido como efeito hormese. Elevados percentuais de mortalidade ocorreram em baixos níveis de concentração, destacando que agentes inseticidas, que normalmente são tóxicos em altos níveis de concentração, apresentaram baixos percentuais de mortalidade (CALABRESE; BALDWIN, 2001). O efeito hormese tem sido relatado em muitos modelos tóxicos para animais e tem sido frequentemente sugerido como o principal mecanismo para o ressurgimento de populações de pragas (CORDEIRO et al., 2013; GUEDES; CUTLER, 2014). Sendo que o intervalo de dose deve ser respeitado para se chegar a uma maior eficiência no manejo, com uma redução da quantidade de produto aplicado.

No entanto, estudos que avaliem a área foliar consumida e a quantificação dos compostos químicos presentes nos extratos se tornam necessários. O que permitiria averiguar os efeitos tóxicos e deterrentes para avaliação da eficácia no campo. Um ponto positivo no manejo de pragas com extratos vegetais é a eficiência de controle em baixas doses, reduzindo os custos de manejo (MIGLIORINI et al., 2010).

Os parâmetros biológicos de $S$. cosmioides e $S$. frugiperda foram afetados pelos extratos utilizados. Os extratos de $A$. macrocarpa e $B$. buttiana tiveram impacto negativo para a qualidade do alimento, proporcionando uma alteração no ganho de peso larval (BOGORNI; VENDRAMIM 2005; RODRIGUES et al., 2008; HAAS et al., 2014). Entretanto, mesmo afetando as características biológicas das lagartas, não foi identificado efeito sobre a viabilidade larval, assim como nos resultados obtido por Moura et al. (2012) em seu experimento com efeito do extrato de $A$. macrocarpa na dieta sobre $S$. frugiperda, com doses variando de $2 \%$ a $6 \%$.

A redução da emergência dos adultos de $S$. cosmioides e $S$. frugiperda implica em efeitos diretos sobre os parâmetros relacionados à reprodução, como período de oviposição, fecundidade, fertilidade e longevidade de machos e fêmeas (SCHMIDT et al., 1997). De acordo com os resultados obtidos, os extratos apresentam efeitos subletais favoráveis ao manejo de pragas, pois reduzem a taxa de crescimento populacional. A redução da viabilidade dos adultos de $S$. frugiperda também foi influenciada pelo extrato de Trichilia spp. (Meliaceae) (ROEL et al., 2000; BOGORNI; VENDRAMIM, 2005), em que a concentração de 60 mg. $L^{-1}$ reduziu em $15 \%$ a viabilidade em relação á testemunha.

Os resultados obtidos nesse trabalho apresentam grande importância no manejo de pragas, como uma alternativa de incremento no manejo, sustentável e eficiente por controlar pragas de ocorrência primária, como $H$. armigera e $S$. frugiperda, e secundária, como S. cosmioides, em cultivos agrícolas.

\section{Agradecimentos}

Agradecemos ao CNPq e à UFPI pelas bolsas de inovação tecnológica concedidas. 


\section{Referências}

ABBASZADEH, A. G.; SRIVASTAVA, C.; WALIA, S. Insecticidal and antifeedant activities of clerodane diterpenoids isolated from the Indian bhant tree, Clerodendron infortunatum, against the cotton bollworm, Helicoverpa armigera. Journal of Insect Science, Madison, v. 14, p. 1-13, 2014.

AHMAD, M. Observed potentiation between pyrethroid and organophosphorus insecticides for the management of Spodoptera litura (Lepidoptera: Noctuidae). Crop Protection, Guildford, v. 28, n. 3, p. 264-268, 2009.

BASKAR, K.; KUMAR, A. S.; RAJ, G. A.; PACKIAM, S. M.; IGNACIMUTHU, S. Bioefficacy of Blumea mollis (D. Don) Merr. and Hygrophila schulii (Buch.-Ham.) (Syn. H. auriculata) against Helicoverpa armigera (Hubner). Archives of Phytopathology and Plant Protection, Philadelphia, v. 48, p. 400-411, 2015.

BASKAR, K.; MAHESWARAN, R.; KINGSLEY, S.; IGNACIMUTHU, S. Bioefficacy of Couroupita guianensis (Aubl) against Helicoverpa armigera (Hub.) (Lepidoptera: Noctuidae) larvae. Spanish Journal of Agricultural Research, Madrid, v. 8, n. 1, p.135-141, 2010.

BOGORNI, P. C.; VENDRAMIM, J. D. Efeito subletal de extratos aquosos de Trichilia spp. sobre o desenvolvimento de Spodoptera frugiperda (J.E. Smith) (Lepidoptera: Noctuidae) em milho. Neotropical Entomology, Londrina, v. 34, n. 2, p. 311-317, 2005. BOIÇA JÚNIOR, A. L.; MEDEIROS, C. A. M.; TORRES, A. L.; FILHO, N. R. C. Efeito de extratos aquosos de plantas no desenvolvimento de Plutella xylostella (1.) (Lepidoptera: Plutellidae) em couve. Arquivos do Instituto Biológico, São Paulo, v. 72, n. 1, p. 45-50, 2005.

CABEZAS, M. F.; NAVA, D. E.; GEISSLER. L. O.; MELO, M.; GARCIA, M. S.; KRÜGER, R. Development and leaf consumption by Spodoptera cosmioides (Walker) (Lepidoptera: Noctuidae) reared on leaves of agroenergy crops. Neotropical Entomology, Londrina, v. 42, n. 6, p. 588-594, 2013.

CALABRESE, E. J.; BALDWIN, L. A. Hormesis: a generalizable and unifying hypothesis. Critical Reviews in Toxicology, London, v. 31, n. 4-5, p. 353-424, 2001.

CHAN, Y. S.; ZHANG, Y.; NG, T. B. Brown kidney bean BowmanBirk trypsin inhibitor is heat and $\mathrm{pH}$ stable and exhibits antiproliferative activity. Applied Biochemistry and Biotechnology, Washington, v. 169, n. 4, p. 1306-1314, 2013.

CORDEIRO, E. M. G.; DE MOURA, I. L. T.; FADINI, M. A. M.; GUEDES, R. N. C. Beyond selectivity: Are behavioral avoidance and hormesis likely causes of pyrethroid-induced outbreaks of the southern red mite Oligonychus ilicis?. Chemosphere, Elmsford, v. 93, n. 6, p. 1111-1116, 2013.

CORONADO, R.; MORRISSETTE, J.; SUKHAREVA, M.; VAUGHAN, D. M. Structure and function of ryanodine receptors. American Journal of Physiology-Cell Physiology, Bethesda, v. 266, n. 6, p. C1485-C1504, 1994.

CZEPAK, C.; ALBERNAZ, K. C.; VIVAN, L. M.; GUIMARÃES, H. O.; CARVALHAIS, T. Primeiro registro de ocorrência de Helicoverpa armigera (Hübner) (Lepidoptera: Noctuidae) no Brasil. Pesquisa Agropecuária Tropical, Goiânia, v. 43, n. 1, p. 110-113, 2013.

DINESH, D. S.; KUMARI, S.; KUMAR, V.; DAS, P. The potentiality of botanicals and their products as an alternative to chemical insecticides to sandflies (Diptera: Psychodidae): a review. Journal of Vector Borne Diseases, New Delhi, v. 5, p. 1-7, 2014.

EL-WAKEIL, N. E. Botanical pesticides and their mode of action. Gesunde Pflanzen, Berlin, v. 65, p. 125-149, 2013.

FIGUEREDO, F. G.; FERREIRA, E. O.; LUCENA, B. F.; TORRES, C. M.; LUCETTI, D. L.; LUCETTI, E. C.; SILVA, J. M. F. L.; SANTOS, F. A. V.; MEDEIROS, C. R.; OLIVEIRA, G. M. M.; COLARES, A. V.; COSTA, J. G. M.; COUTINHO, H. D. M.; MENEZES, I. R. A.; SILVA, J. C. F.; KERNTOPF, M. R.; FIGUEIREDO, P. R. L.; MATIAS, E. F. F. Modulation of the antibiotic activity by extracts from Amburana cearensis AC Smith and Anadenanthera macrocarpa (Benth.) Brenan. BioMed Research International, New York, v. 2013, 2013.

GUEDES, R. N. C.; CUTLER, G. C. Insecticide-induced hormesis and arthropod pest management. Pest Management Science, Malden, v. 70, p. 690-697, 2014.

HAAS, J.; GARCIA, B. C.; ALVES, L. F. A.; HAIDA, K. S. Efeito de extratos aquosos vegetais sobre a lagarta-do-cartucho. Arquivos do Instituto Biológico, São Paulo, v. 81, n. 1, p. 79-82, 2014.

KASTEN JR., P.; PRECETTI, A. A. C. M.; PARRA, J. R. P. Dados biológicos comparativos de Spodoptera frugiperda (J. E. Smith, 1797) em duas dietas artificiais e substrato natural. Revista Agricultura, Piracicaba, v. 53, n. 1/2, p. 68-78, 1978.

KATSUDA, Y. Development of and future prospects for pyrethroid chemistry. Pest Management Science, Malden, v. 55, n. 8, p. $775-$ $782,1999$.

LEITE, N. A.; MENDES, S. M.; SANTOS-AMAYA, O. F.; SANTOS, C. A.; TEIXEIRA, T. P.; GUEDES, R. N.; PEREIRA, E. J. Rapid selection and characterization of CrylF resistance in a Brazilian strain of fall armyworm. Entomologia Experimentalis et Applicata, Dordrecht, v. 158, n. 3, p. 236-247, 2016.

LODHA, M. L.; CHOUDHARY, N. L.; MAHAPATRO, G. K.; SINGH, B.; GUPTA, G. P. Purification and evaluation of antiviral proteins from Bougainvillea $\mathrm{x}$ buttiana against Helicoverpa armigera. Indian Journal of Agricultural Sciences, New Delhi, v. 81, n. 1, p. 74-78, 2011.

MEDEIROS, C. A. M.; BOIÇA JUNIOR, A. L.; TORRES, A. L. Efeito de extratos aquosos de plantas na oviposição da traça-dascrucíferas, em couve. Bragantia, Campinas, v. 64, n.2, p. 227-232, 2005.

MIGLIORINI, P.; LUTINSKI, J. A.; GARCIA, F. R. M. Eficiência de extratos vegetais no controle de Diabrotica speciosa (Germar, 1824) (Coleoptera: Chrysomelidae), em laboratório. Biotemas, Florianópolis, v. 23, n. 1, p. 83-89, 2010.

MORI, S. A.; SILVA, L. A. M.; LISBOA, G.; CORADIN, L. Manual de manejo de herbário fanerogâmico. 2. ed. Ilhéus: Centro de Pesquisas do Cacau, 1989. p. 104.

MOURA, J. Z.; PÁDUA, L. E. de M.; E SILVA, P. R. R.; SILVA, A. A.; MAGGIONI, K. Extrato de folhas de Anadenanthera macrocarpa sobre a biologia de Spodoptera frugiperda criada em dieta artificial. Comunicata Scientiae, Bom Jesus, v. 3, n. 4, p. 249-254, 2012.

PARRA-GARCÉS, M. I.; CAROPRESE-ARAQUE, J. F.; ARRIETA-PRIETO, D.; STASHENKO, E. Morfología, anatomía, ontogenia y composición química de metabolitos secundarios en inflorescencias de Lippia alba (Verbenaceae). Revista de Biologia Tropical, San José, v. 58, n. 4, p. 1533-1548, 2010. 
R CORE TEAM. R: a language and environment for Statiscarica Compunting. 2015. Vienna: R. Foundation for Statistical Computing. Disponível em: <http://www.R-project.org/>.

RIBEIRO, A. O.; SILVA, A. F.; CASTRO, A. H. F. Identificação de espécies da família Asteraceae, revisão sobre usos e triagem fitoquímica do gênero Eremanthus da Reserva Boqueirão, IngaíMG. Revista Brasileira de Plantas Medicinais, Botucatu, v. 4, n. 12, p. 456-65, 2010.

RODRIGUES, S. R.; COUTINHO, G. V.; GARCEZ, W. S.; GARCEZ, F. R.; ZANELLA, D. P. de F. Atividade inseticida de extratos etanólicos de plantas sobre Spodoptera frugiperda (J.E.Smith) (Leptopdera: Noctuidae). Agrarian, Dourados, v. 1, n. 1, p. 133-144, 2008.

ROEL, A. R.; VENDRAMIM, J. D.; FRIGHETTO, R. T. S.; FRIGHETTO, N. Efeito do extrato acetato de etila de Trichilia pallida Swartz (Meliaceae) no desenvolvimento e sobrevivência da lagarta-do-cartucho. Bragantia, Campinas, v. 59, n. 1, p. 53-58, 2000

SAS INSTITUTE. SAS user's manual, version 9.1. 2002. Disponível em: <https://support.sas.com/documentation/ onlinedoc/91pdf/sasdoc_91/stat_ug_7313.pdf $>$.

SCHMIDT, G. H.; AHMED, A. A. I.; BREUER, M. Effect of Melia azedarach extract on larval development and reproduction parameters of Spodoptera littoralis (Boisd.) and Agrotis ipsilon (Hufn.) (Lep. Notuidae). Anzeiger für Schädlingskunde, Pflanzenschutz, Umweltschutz, Berlin, v. 70, p. 4-12, 1997.

SILVA, W.; FREIRE, M. das. G. M.; PARRA, J. R. P.; S. MARANGONI; MACEDO, M. L. R. Evaluation of the Adenanthera pavonina seed proteinase inhibitor (ApTI) as a bioinsecticidal tool with potential for the control of Diatraea saccharalis. Process Biochemistry, Amsterdam, v. 47, p. 257-263, 2012.
SILVA FILHO, M. L.; SILVA, L. B.; FERNANDES, R. M.; LOPES, G. S. Efeito do extrato aquoso e etanólico do angico preto sobre larvas de Rhipicephalus (Boophilus) microplus. Arquivo Brasileiro de Medicina Veterinária e Zootecnia, Minas Gerais, v. 65, n. 3, p. 637-644, 2013.

TRIGGLE, D. J.; MITCHELL, J. M.; FILLER, R. The pharmacology of physostigmine. CNS Drug Reviews, San Francisco, v. 4, n. 2, p. 87-136, 1998.

VARMA, J.; DUBEY, N. K. Prospectives of botanical and microbial products as pesticides of tomorrow. Current Science, Bangalore, $v$. 76, n. 2, p. 172-179, 1999

VIEGAS-JÚNIOR, C. Terpenos com atividade inseticida: uma alternativa para o controle químico de insetos. Química Nova, São Paulo, v. 26, n. 3, p. 390-400, 2003.

WANG, K. Y.; ZHANG, Y.; WANG, H. Y.; XIA, X. M.; LIU, T. $\mathrm{X}$. Influence of three diets on susceptibility of selected insecticides and activities of detoxification esterases of Helicoverpa assulta (Lepidoptera: Noctuidae). Pesticide Biochemistry and Physiology, San Diego, v. 96, n. 1, p. 51-55, 2010.

WEBER, C. R.; SOARES, C. M.; LOPES, A. B.; SILVA, T. S.; NASCIMENTO, M. S.; XIMENES, E. C. Anadenanthera colubrina: um estudo do potencial terapêutico. Revista Brasileira de Farmácia, Rio de Janeiro, v. 92, n. 4, p. 235-244, 2011.

ZHAO, B.; MOOCHHALA, S. M.; THAM, S-Y. Biologically active components of Physostigma venenosum. Journal of Chromatography B, Tallahassee, v. 812, n. 1, p. 183-192, 2004. 